\title{
IDENTIFICAÇÃO E CONTROLE DE PROCESSOS VIA DESENVOLVIMENTOS EM SÉRIES ORTONORMAIS. PARTE B: CONTROLE PREDITIVO
}

\author{
Gustavo H. C. Oliveira* \\ gustavo.oliveiraepucpr.br \\ Wagner C. Amaral ${ }^{\ddagger}$ \\ wagner@dca.fee.unicamp.br \\ *PPGEPS/CCET/PUCPR \\ Rua Imaculada Conceição 1155 CEP 80215-901 \\ Curitiba - PR, Brasil \\ ${ }^{\dagger} \mathrm{SCC} / \mathrm{ICMC} / \mathrm{USP}$ \\ CP 668 CEP 13560-970 \\ São Carlos - SP, Brasil \\ ${ }^{\ddagger}$ DCA/FEEC/UNICAMP \\ CP 6101 CEP 13083-970 \\ Campinas - SP, Brasil
}

Ricardo J. G. B. Campello ${ }^{\dagger}$

campellodicmc.usp.br

\begin{abstract}
This paper presents an overview about predictive control schemes based on orthonormal basis function models. Different predictive control schemes based on such models are discussed, namely, linear controllers with terminal (stabilizing) constraints, robust controllers, and non-linear controllers. The discussions comprise a broad bibliographical survey on the subject as well as two case studies involving a simulated dynamic system and a real process.
\end{abstract}

KEYWORDS: Orthonormal Basis Functions, Predictive Control, Non-Linear Control, Robust Control.

\section{RESUMO}

O presente artigo aborda o problema da seleção da estrutura de modelos em algoritmos de controle preditivo para sistemas monovariáveis. Neste sentido, apresenta a utilização de modelos com estrutura dinâmica desenvolvida através de bases de funções ortonormais, como as funções de Laguerre, Kautz ou funções ortonormais generalizadas. Os principais aspectos relacionados com esta classe de modelos no contexto de controladores preditivos lineares com restrições terminais, não lineares e robusto são discutidos e uma revisão bibliográfica é apresentada. O desempenho de malha fechada das estratégias analisadas é ilustrado através de dois casos de estudo envolvendo uma incubadora para recém nascidos e um processo simulado de polimerização isotérmica.

PALAVRAS-CHAVE: Bases de Funções Ortonormais, Controle Preditivo, Controle de Sistemas Não Lineares, Controle Robusto.

\footnotetext{
Artigo submetido em 14/08/2006

1a. Revisão em 19/03/2007

Aceito sob recomendação do Editor Associado

Prof. Liu Hsu
} 


\section{INTRODUÇÃO}

Controladores do tipo preditivo (ou Model Based Predictive Controllers - MBPC) tiveram sua origem na década de 70 e, desde então, são amplamente analisados na literatura de controle. Atualmente, é bem conhecido que algoritmos de controle preditivo podem ser utilizados no controle de uma ampla gama de processos, dentre eles os processos instáveis, de fase não mínima, não lineares, com longo atraso de transporte, híbridos, etc, sem mudanças significativas do ponto de vista conceitual (Soeterboek, 1992; Clarke, 1994; Camacho and Bordons, 1999; Allgower and Zheng, 2000; Rossiter, 2003). Podem incorporar, na lei de controle, restrições operacionais relacionadas com os sinais de entrada / saída e possuem parâmetros de sintonia de fácil ajuste. Também podem ser reparametrizados como controladores do tipo modelo interno ou Internal Model Controllers - IMC (Garcia et al., 1989). Devido a todos estes aspectos, atualmente os MBPC não estão restritos somente ao meio acadêmico e são conhecidas diversas aplicações na indústria de processos (Qina and Badgwell, 2003), com destaque para o setor químico e petroquímico, e.g. (Prett and Garcia, 1988; Prett and Garcia, 1989).

Os MBPC são definidos a partir do modelo do processo que se deseja controlar e são caracterizados por quatro etapas principais, descritas a seguir:

i) Modelagem do processo. Equações de previsão da saída são utilizadas para prever o comportamento do processo em um horizonte de tempo futuro, denominado horizonte de previsão.

ii) Definição de um critério de custo. O desempenho do sistema em malha fechada durante o horizonte de previsão é especificado através de um critério de custo, definido a partir da saída prevista, do sinal de referência e do esforço de controle.

iii) Otimização do critério de custo. O critério de custo é minimizado em relação ao conjunto de futuros sinais de controle (em um horizonte de tempo denominado de horizonte de controle) a serem aplicados no processo.

iv) Utilização do sinal de controle ótimo. Somente o primeiro sinal de controle resultante da otimização do critério de custo é realmente utilizado no processo. No instante seguinte de amostragem, todo o procedimento é repetido (receding horizon control strategy).

A estrutura aberta e genérica deste esquema para controladores MBPC permitiu a origem de muitos algoritmos presentes na literatura As principais diferenças entre as abordagens MBPC já propostas residem na maneira pela qual cada uma destas etapas citadas é formulada.
No que diz respeito ao critério de custo, este pode ser implementado utilizando-se as normas 1,2 ou $\infty$ (Campo and Morari, 1986). Já no que diz respeito à otimização do critério de custo, os trabalhos iniciais na área realizam otimização sem restrições, e.g. (Clarke et al., 1987; de Keyser et al., 1988). Na seqüência, restrições são incluídas para lidar com limitações nos atuadores e/ou na saída (Tsang and Clarke, 1988; Camacho, 1993). Na década de 90, em trabalhos como (Clarke and Scattolini, 1991; Mosca and Zhang, 1992; Mayne et al., 2000), restrições terminais são incluídas no critério de custo para garantir estabilidade do sistema em malha fechada quando o horizonte de previsão é finito.

Adicionalmente, a realização da lei de controle no que diz respeito ao tratamento do modelo do processo deu origem também a diferentes tipos de controle preditivos, por exemplo, adaptativo (de Keyser et al., 1988; Dumont et al., 1993), robusto (Zheng and Morari, 1993; Kothare et al., 1996; Oliveira et al., 2000), múltiplos modelos (Chow et al., 1995; Trierweiler and Secchi, 2000), ganho escalonado (Zhu et al., 1991), etc.

Um importante ponto relacionado com algoritmos MBPC é a escolha do modelo do processo para cálculo da previsão da saída, uma vez que este é determinante para o desempenho do sistema em malha fechada. O projeto de um algoritmo de controle, baseado em um modelo inadequado, pode não resultar no desempenho especificado quando aplicado no controle do processo real.

Por outro lado, a utilização de funções ortonormais na modelagem e identificação de processos teve um grande impulso com trabalhos como (den Hof et al., 1995; Wahlberg and Makila, 1996; Heuberger et al., 2005), dentre outros (e.g. veja (Patwardhan and Shah, 2005; Patwardhan et al., 2006) e referências inclusas). As propriedades deste tipo de representação de sistemas lineares e não lineares, i.e., (non-linear) othornormal basis functions models - (N)OBF, é apresentada na primeira parte deste artigo (Campello et al., n.d.). Devido ao fato dos algoritmos de controle preditivo permitirem a utilização de diferentes estruturas de modelo, este esquema de controle é uma aplicação para modelos (N)OBF. Neste contexto se inclui a escolha de bases de funções ortonormais como estrutura de modelo de processos dinâmicos para formulação de equações de previsão em algoritmos MBPC. Apesar de aplicações de bases de funções ortonormais na representação de sistemas multivariáveis estarem presentes na literatura, este artigo possui foco no problema de controle monovariável.

O presente artigo apresenta os principais aspectos relativos à utilização de modelos (N)OBF em algoritmos de controle preditivo e está estruturado como se segue. Na Seção 2, uma 
revisão dos modelos utilizados em algoritmos de controle preditivo é apresentada. Nas Seções 3, 4 e 5, as propriedades dos algoritmos de controle com modelos lineares, não lineares e com incertezas paramétricas (robusto), respectivamente, são descritas. Na Seção 6, exemplos ilustram características de alguns dos algoritmos descritos e, finalmente, na Seção 7, o artigo é concluído.

\section{MODELOS UTILIZADOS EM CON- TROLE PREDITIVO}

Sob o ponto de vista do modelo utilizado para representação do processo e cálculo da previsão da saída, pode-se classificar algoritmos MBPC em estratégias que utilizam modelos dinâmicos lineares, modelos dinâmicos não lineares ou modelos híbridos (dinâmica e lógica). Estes modelos também podem ser descritos através de parâmetros constantes, variantes no tempo, incertos ou não.

Os algoritmos de controle preditivo com modelos lineares podem ser separados em duas classes principais, aqueles com modelos estruturados, como os modelos Auto Regressive Integrated Moving Average with Exogenous Input - AR(I)MAX e similares, e os com modelos não estruturados, como os modelos Finite Impulse Response - FIR ou OBF. Estas duas classes de modelos podem ser reescritas no formato de espaço de estados.

Neste sentido, os primeiros algoritmos de controle preditivo com modelo linear propostos na literatura tiveram como ponto de partida equações de previsão baseadas na resposta ao impulso (modelos FIR) ou, de forma equivalente, na resposta ao degrau. Os casos mais conhecidos neste contexto são o Model Adaptive Controller - MAC e o Dynamic Matrix Controller - DMC (Richalet et al., 1978; Cutler and Ramaker, 1980). Estes algoritmos, bastante difundidos no controle de processos químicos e petro-químicos, também apresentam formulação baseada em espaço de estados, conforme descrito em (Li et al., 1989; Lee et al., 1994).

Os modelos lineares de estrutura AR(I)MAX e similares estão presentes nos algoritmos Generalized Predictive Controller - GPC (Clarke and Mohtadi, 1989), Extended Predictive Self-Adaptive Controller - EPSAC (de Keyser and Cuawenberghe, 1985), Extended Horizon Predictive Controller - EHPC (Ydstie, 1984), dentre outros (veja detalhes em (de Keyser et al., 1988; Soeterboek, 1992; Camacho and Bordons, 1999)). Em (Soeterboek, 1992) propõe-se o UPC Unified Predictive Controller, que unifica, em uma só estrutura, diferentes representações lineares para o modelo de previsão da lei de controle. A representação do processo usando um modelo linear ARIMAX deu origem ao GPC, que é um dos mais conhecidos esquemas MBPC. Adicionalmente, algoritmos com modelos em espaço de estados base- ados neste esquema são propostos, por exemplo, em (Ordys and Clarke, 1993). Representações em espaço de estados usualmente requerem um estimador de estados. O desenvolvimento de equações de previsão neste contexto é explorada em (Ordys and Clarke, 1993; Camacho and Bordons, 1999).

Algoritmos de controle preditivo baseados em modelos de resposta ao impulso ou degrau são aplicáveis somente em processos estáveis em malha aberta. Usualmente, os modelos possuem uma grande quantidade de parâmetros, o que dificulta aplicações em controle adaptativo. Por outro lado, a seleção da estrutura (ordem e atraso de transporte) de modelos AR(I)MAX representa uma tarefa adicional na fase de identificação de sistemas.

Modelos lineares formados por funções ortonormais podem ser vistos como uma extensão dos modelos de resposta ao impulso. Sua utilização no contexto de controle preditivo é apresentada em (Zervos and Dumont, 1988; Finn et al., 1993; Elshafei et al., 1994; Oliveira et al., 1999). Basicamente, as mesmas propriedades dos algoritmos MBPC com modelos de resposta ao impulso / degrau são observadas quando modelos OBF são utilizados. Uma vantagem importante deste último é a maior parcimônia em termos de número de parâmetros na representação do processo. Dentre os exemplos de aplicações industriais, tem-se (Dumont et al., 1990) dentre outros. O modelo OBF também admite representação em espaço de estados como nos outros casos abordados anteriormente. Entretanto, todos os estados são conhecidos pois, devido à estrutura do modelo, podem ser calculados através do sinal de entrada; portanto, não há necessidade de implementação de um observador de estados. Assim como os modelos de resposta ao impulso, controladores preditivos baseados em modelos OBF são aplicáveis em sistemas estáveis em malha aberta com ou sem ação integral.

Muitas vezes, a hipótese de linearidade não é verificada na prática, pois a maioria dos processos reais contêm, em algum grau, não linearidades. Com esta motivação, alguns algoritmos MBPC baseados em modelos não lineares foram propostos na literatura (Allgower and Zheng, 2000), por exemplo, MBPC com modelos baseados em redes neurais (SaintDonat, 1991) ou sistemas fuzzy (Oliveira and Lemos, 1995) e MBPC com modelos Hammerstein (Zhu et al., 1991; Haber et al., 1998), Wiener (Norquay et al., 1999; Wellers and Rake, 2000) e Volterra (Doyle et al., 1995; Dolye et al., 2001; Oliveira et al., 2003).

Diferentes estruturas e realizações de modelos não lineares são conhecidas na literatura; por exemplo, as estruturas NAR(MA)X, NOE, NFIR, NOBF, Wiener e Hammerstein (Ljung, 1999; Aguirre, 2000; Nelles, 2001; Campello and Oliveira, 2007). Como realizações, pode-se citar os mapeamentos Racionais, Polinomiais, Fuzzy, com Redes Neurais, 
com Séries de Volterra, etc. As estruturas Wiener e Hammerstein, em geral, são dependentes de não linearidades específicas do processo. Já as estruturas NAR(MA)X e NOE, de forma análoga ao caso linear, necessitam de uma escolha associada com o regressor utilizado no modelo do processo, isto é, os termos passados relevantes dos sinais de entrada e saída do processo. As estruturas NFIR e NOBF possuem a vantagem de não requerer uma definição explícita deste regressor. Entretanto, um número elevado de parâmetros muitas vezes é necessário no caso NFIR. A estrutura NOBF dá origem a modelos com menos parâmetros e mesma qualidade de representação que uma mesma realização do tipo NFIR.

Modelos com incerteza nos parâmetros também podem ser utilizados em algoritmos de controle preditivo, dando origem aos denominados algoritmos de controle preditivo robusto, ou RMBPC. Neste contexto, as primeiras propostas de controle preditivo robusto, baseados na obtenção da lei de controle via solução de um problema de otimização min-max, utilizavam modelos de resposta ao impulso/degrau (Campo and Morari, 1987; Biegler, 1989; Zheng and Morari, 1993). Nesta abordagem, a equação de previsão deve ser afim em relação ao vetor que representa a incerteza nos parâmetros para permitir a solução numérica do problema de otimização. Algoritmos utilizando modelos de resposta ao impulso e ao degrau com coeficientes incertos foram desenvolvidos para o controle do processo benchmark Shell, como por exemplo, o descrito em (Cuthrell et al., 1989; Biegler, 1989). A utilização de modelos ARIMAX para a modelagem de processos incertos neste contexto de RMBPC é descrita em (Gutierrez and Camacho, 1995). Esta proposta, apesar de gerar um espaço de incertezas de menor dimensão (menos parâmetros incertos) que os modelos FIR incertos, leva a uma equação de previsão que não é afim ao vetor de incertezas, impedindo a obtenção de uma solução ótima para o problema de controle.

A utilização da estrutura OBF na representação de modelos incertos gera um espaço de incertezas de dimensão menor que o gerado pela estrutura FIR. Como consequiência, tem-se um menor custo computacional do problema de otimização e a equação de previsão obtida é afim em relação ao vetor de incertezas, garantindo a existência de solução numérica da lei de controle.

Outra abordagem de controle preditivo robusto está baseada na utilização de modelos por espaço de estados com parâmetros incertos, onde incerteza está muitas vezes representada na forma de politopos. Uma característica destes algoritmos é a representação da lei de controle através de LMIs, como em (Kothare et al., 1996; Cuzzola et al., 2002; Ding. et al., 2004). Uma vez que são baseados em realimentação de estados, estes métodos freqüentemente requerem um estimador de estados.
Modelos híbridos são modelos que aliam, em uma descrição única, variáveis em tempo contínuo e variáveis a eventos discretos, isto é, dinâmica e lógica (Labinaz et al., 1997; Lennartson et al., 1994; Heemels et al., 2001). Algoritmos de controle preditivo com modelos híbridos são descritos em (Bemporad and Morari, 1999; Bemporad et al., 2000; Malacalza and Jr., 2005). Em (Moreira, 2006), a utilização da estrutura OBF é proposta neste contexto, para os casos com e sem incerteza nos parâmetros do modelo.

\section{CONTROLE PREDITIVO COM MODE- LOS LINEARES}

A modelagem de sistemas lineares estáveis utilizando bases ortonormais é usualmente realizada através da seguinte representação em espaço de estados:

$$
\begin{cases}\boldsymbol{l}(k+1) & =A \boldsymbol{l}(k)+\boldsymbol{b} u(k) \\ y(k) & =\boldsymbol{c}^{T} \boldsymbol{l}(k)\end{cases}
$$

Nesta representação, cada sinal $l_{i}(k)$, que compõe o vetor de estados $\boldsymbol{l}(k)$, é a saída do $i$-ésimo filtro ortonormal para a entrada $u(k)$. Nesta representação, os elementos das matrizes $A$ e $\boldsymbol{b}$ são calculados a partir da base ortonormal selecionada e de seus parâmetros. Suas dimensões são definidas em função da ordem de truncamento $n$ da representação em séries do processo (Heuberger et al., 2005; Campello et al., n.d.).

Os elementos $c_{i}, i=1, \ldots, n$, presentes no vetor $c$, representam a parametrização do processo em relação a uma base de funções previamente selecionada. Todos os estados do modelo podem ser calculados em função da entrada $u(k)$. Esta representação em espaço de estados, reescrita a seguir, é o ponto de partida para a formulação de equações de previsão, etapa necessária para a síntese da lei de controle. Reescrevendo o modelo (1) no formato incremental, obtém-se:

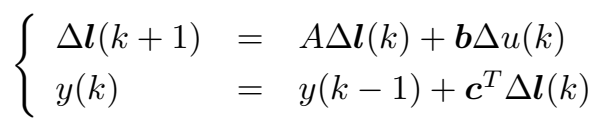

onde $\Delta$ é o operador $1-q^{-1}$. Através deste modelo, a seguinte previsão da saída $j$ passos a frente, i.e. $\hat{y}(k+j \mid k)$, pode ser construída:

$$
\hat{y}(k+j \mid k)=\boldsymbol{c}^{T} \sum_{m=1}^{j} S_{j-m} \boldsymbol{b} \Delta u(k+m-1)+\hat{y}_{l}(k+j \mid k)
$$

$\mathrm{e}$

$$
\hat{y}_{l}(k+j \mid k)=y(k)+\boldsymbol{c}^{T} S_{j-1} A \Delta \boldsymbol{l}(k)
$$


sendo $S_{j}=\sum_{i=0}^{j} A^{j}$.

O algoritmo de controle preditivo é obtido através da minimização de um critério de custo em relação aos futuros valores do sinal de controle. Diferentes normas podem ser utilizadas na formulação do critério de custo, entretanto, a norma Euclidiana é a mais usual. Assim sendo, o critério $J_{k}$ é dado por:

$J_{k}=\sum_{j=1}^{N_{y}}(\hat{y}(k+j \mid k)-w(k+j))^{2}+\sum_{j=0}^{N_{u}-1} \lambda \Delta u^{2}(k+j \mid k)$

onde $w(k)$ é a referência desejada e $\lambda$ um fator de ponderação para o esforço de controle. A previsão da saída é realizada durante o horizonte de previsão $N_{y}$. O horizonte de controle $N_{u}$ limita o grau de liberdade de futuras variações no sinal de controle. A lei de controle é, portanto, definida pelo seguinte problema de otimização:

$$
\begin{gathered}
\min _{\Delta u(k), \ldots, \Delta u\left(k+N_{u}-1\right)} J_{k} \\
\text { s.a } \\
\Delta u(k+j \mid k)=0 \quad \forall j=N_{u}, \ldots, N_{y} \\
\hat{y}\left(k+N_{y}+j \mid k\right)=w\left(k+N_{y}+j\right) \quad \forall j=1, \ldots, m
\end{gathered}
$$

com $0<m<N_{u}$. Neste problema de otimização, a função objetivo é o critério de custo. O primeiro conjunto de restrições garante o horizonte de controle, i.e., que implica em variação nula do sinal de controle após determinado instante de tempo futuro. O segundo conjunto de restrições são aplicadas nos valores imediatamente após o horizonte de previsão para garantia de estabilidade do sistema em malha fechada, conforme discutido a seguir.

Uma dificuldade encontrada em muitos algoritmos MBPC com modelos lineares é o fato que os resultados sobre a estabilidade do sistema em malha fechada estão ligados ao uso de horizontes e ponderações infinitos no critério de custo (Clarke and Mohtadi, 1989). Esta linha é explorada em trabalhos como (Bitmead et al., 1990). A busca da solução para este problema levou ao desenvolvimento de novos algoritmos, dentre eles, o Constrained Receding Horizon Predictive Controller (CRHPC) (Clarke and Scattolini, 1991). Este tipo de algoritmo surgiu como uma alternativa à abordagem clássica, através da inclusão de restrições nos valores terminais da previsão da saída, uma vez que a estabilidade do sistema em malha fechada é garantida através de condições menos restritivas em relação à formulação clás- sica. Na formulação original do CRHPC, apresentada em (Clarke and Scattolini, 1991), o horizonte de controle é igual ao horizonte de previsão. Posteriormente, em (Leva and Scattolini, 1993), o caso geral (ie, $N_{u} \neq N_{y}$ ) é incluído no algoritmo. Na mesma linha de desenvolvimento, em (Demircioglu and Clarke, 1993) propõe-se a incorporação, no critério de custo, de uma ponderação no valor final dos estados do processo, dando origem ao Generalized Predictive Control Weighting (GPCW). Este algoritmo pode ser visto como um controlador que engloba o GPC e o CRHPC, onde o primeiro é obtido com uma ponderação nula nos estados e o segundo com uma ponderação infinita nos estados.

Por outro lado, usualmente restrições de amplitude e variação no sinal de controle e de amplitude do sinal de saída, i.e.,

$$
\begin{gathered}
u_{\min } \leq u(k+j-1 \mid k) \leq u_{\max } \quad \forall j=1, \ldots, N_{u} \\
\Delta u_{\min } \leq \Delta u(k+j-1 \mid k) \leq \Delta u_{\max } \quad \forall j=1, \ldots, N_{u} \\
y_{\min } \leq \hat{y}(k+j \mid k) \leq y_{\max } \quad \forall j=1, \ldots, N_{y}
\end{gathered}
$$

são também incorporadas à lei de controle para lidar com restrições operacionais do processo. A solução da lei de controle com estas restrições operacionais possui somente solução numérica (através de um algoritmo de programação quadrática). Entretanto, na ausência destas restrições, a lei de controle possui solução analítica conforme apresentado a seguir, para o caso onde $w(k+j)=w(k), j=1, \ldots, N_{y}$.

O problema de otimização (6), reescrito de uma forma vetorial é dado por:

$$
\begin{gathered}
\min _{\substack{\Delta \boldsymbol{u} \\
\text { s.a }}}(\hat{\boldsymbol{y}}-\boldsymbol{w})^{T}(\hat{\boldsymbol{y}}-\boldsymbol{w})+\lambda \Delta \boldsymbol{u}^{T} \Delta \boldsymbol{u} \\
\hat{\boldsymbol{y}}_{c}=\boldsymbol{w}_{c}
\end{gathered}
$$

onde $\hat{\boldsymbol{y}}$ e $\hat{\boldsymbol{y}}_{c}$ são vetores cujos elementos são as previsões da saída (ver equação (3)) para $j=1, \ldots, N_{y}$ e $j=N_{y}+1, \ldots, N_{y}+m$, respectivamente. $\boldsymbol{w}$ e $\boldsymbol{w}_{c}$ são vetores de dimensão apropriada cujos elementos são iguais a $w(k)$. Além disso, $\hat{\boldsymbol{y}}$ e $\hat{\boldsymbol{y}}_{c}$ são respectivamente dados por:

$$
\begin{gathered}
\hat{\boldsymbol{y}}=H \Delta \boldsymbol{u}+\hat{\boldsymbol{y}}_{l} \\
\hat{\boldsymbol{y}}_{c}=H_{c} \Delta \boldsymbol{u}+\hat{\boldsymbol{y}}_{l c}
\end{gathered}
$$

onde

$$
H=\left[\begin{array}{cccc}
\boldsymbol{c}^{T} S_{0} \boldsymbol{b} & 0 & 0 & \cdots \\
\boldsymbol{c}^{T} S_{1} \boldsymbol{b} & \boldsymbol{c}^{T} S_{0} \boldsymbol{b} & 0 & \\
\boldsymbol{c}^{T} S_{2} \boldsymbol{b} & \boldsymbol{c}^{T} S_{1} \boldsymbol{b} & \boldsymbol{c}^{T} S_{0} \boldsymbol{b} & \\
\vdots & & &
\end{array}\right]_{N_{y} \times N_{u}}
$$




$$
H_{c}=\left[\begin{array}{ccc}
\boldsymbol{c}^{T} S_{N_{y}} \boldsymbol{b} & \boldsymbol{c}^{T} S_{N_{y}} \boldsymbol{b} & \ldots \\
\boldsymbol{c}^{T} S_{N_{y}+1} \boldsymbol{b} & \boldsymbol{c}^{T} S_{N_{y}} \boldsymbol{b} & \\
\vdots & \vdots & \\
\boldsymbol{c}^{T} S_{N_{y}+m-1} \boldsymbol{b} & \boldsymbol{c}^{T} S_{N_{y}} \boldsymbol{b} & \ldots
\end{array}\right]_{m \times N_{u}}
$$

e $\hat{\boldsymbol{y}}_{l}$ e $\hat{\boldsymbol{y}}_{l c}$ são vetores cujos elementos são as previsões livre da saída (ver equação (4)) para $j=1, \ldots, N_{y}$ e $j=N_{y}+$ $1, \ldots, N_{y}+m$, respectivamente.

O vetor de futuras ações de controle, solução do problema de otimização (8), é dado por (Clarke and Scattolini, 1991):

$$
\begin{gathered}
\Delta \boldsymbol{u}=Q^{-1}\left(I-H_{c}^{T}\left(H_{c} Q^{-1} H_{c}^{T}\right)^{-1} H_{c} Q^{-1}\right) H^{T} \\
\times\left(\boldsymbol{w}-\hat{\boldsymbol{y}}_{l}\right)+Q^{-1} H_{c}^{T}\left(H_{c} Q^{-1} H_{c}^{T}\right)^{-1}\left(\boldsymbol{w}_{c}-\hat{\boldsymbol{y}}_{l c}\right)
\end{gathered}
$$

onde $Q=\left(H^{T} H+\lambda I\right)$. Neste vetor, o primeiro elemento, i.e. $\Delta u(k)$, é o valor efetivamente utilizado no controle do processo, com $u(k)=u(k-1)+\Delta u(k)$. No próximo instante de amostragem, todo o procedimento é repetido. Portanto:

$$
\Delta u(k)=\boldsymbol{n}^{T}\left(\boldsymbol{w}-\hat{\boldsymbol{y}}_{l}\right)+\boldsymbol{p}^{T}\left(\boldsymbol{w}_{c}-\hat{\boldsymbol{y}}_{l c}\right)
$$

onde

$$
\begin{aligned}
\boldsymbol{n}^{T}=\left[\begin{array}{llll}
1 & 0 & \cdots & 0
\end{array}\right] Q^{-1}\left(I-H_{c}^{T}\left(H_{c} Q^{-1} H_{c}^{T}\right)^{-1}\right. \\
\left.\times H_{c} Q^{-1}\right) H^{T} \\
\boldsymbol{p}^{T}=\left[\begin{array}{llll}
1 & 0 & \cdots & 0
\end{array}\right] Q^{-1} H_{c}^{T}\left(H_{c} Q^{-1} H_{c}^{T}\right)^{-1}
\end{aligned}
$$

Sejam $G, G_{c}, n_{\mathrm{s}}, p_{\mathrm{s}}$ e $e(k)$ :

$$
\begin{gathered}
G=\left[\begin{array}{c}
\boldsymbol{c}^{T} S_{0} A \\
\boldsymbol{c}^{T} S_{1} A \\
\vdots \\
\boldsymbol{c}^{T} S_{N_{y}-1} A
\end{array}\right] \\
G_{c}=\left[\begin{array}{c}
\boldsymbol{c}^{T} S_{N_{y}} A \\
\boldsymbol{c}^{T} S_{N_{y}+1} A \\
\vdots \\
\boldsymbol{c}^{T} S_{N_{y}+m-1} A
\end{array}\right] \\
e(k)=w(k)-y(k) \\
n_{\mathrm{s}}=\sum_{i=1}^{N_{y}} n_{i}
\end{gathered}
$$

$\mathrm{e}$

$$
p_{\mathrm{s}}=\sum_{i=1}^{m} p_{i}
$$

onde $n_{i}$ e $p_{i}$ são as $i$-ésimas componentes dos vetores $\boldsymbol{n}$ e $\boldsymbol{p}$, respectivamente.

A partir destas definições e da equação (2), obtém-se:

$$
\left\{\begin{array}{l}
\Delta \boldsymbol{l}(k+1)=A \Delta \boldsymbol{l}(k)+\boldsymbol{b} \Delta u(k) \\
\Delta u(k)=-\left(\boldsymbol{n}^{T} G+\boldsymbol{p}^{T} G_{c}\right) \Delta \boldsymbol{l}(k)+\left(n_{\mathrm{s}}+p_{\mathrm{s}}\right) e(k)
\end{array}\right.
$$

ou

$$
\left\{\begin{array}{c}
\Delta \boldsymbol{l}(k+1)=\left[A-\boldsymbol{b}\left(\boldsymbol{n}^{T} G+\boldsymbol{p}^{T} G_{c}\right)\right] \Delta \boldsymbol{l}(k) \\
+\left[\boldsymbol{b}\left(n_{\mathrm{s}}+p_{\mathrm{s}}\right)\right] e(k) \\
\Delta u(k)=-\left(\boldsymbol{n}^{T} G+\boldsymbol{p}^{T} G_{c}\right) \Delta \boldsymbol{l}(k)+\left(n_{\mathrm{s}}+p_{\mathrm{s}}\right) e(k)
\end{array}\right.
$$

Uma vez que o modelo com bases ortonormais é estável, observável e alcançável, todas as propriedades estabilizantes do controlador CRPHC, descritas em (Clarke and Scattolini, 1991), podem ser aplicadas.

Algoritmos de controle preditivo baseados em modelos construídos com funções ortonormais apresentam características semelhantes aos baseados em modelos FIR, e.g. o DMC ou QDMC e, como estes, não são aplicáveis em sistemas instáveis em malha aberta. Não há necessidade de estimação de estados uma vez que estes são calculados diretamente a partir do sinal de entrada, nem da determinação da ordem do modelo. A seleção adequada de bases ortonormais na construção do modelo permite representar o modelo com uma redução significativa do número de parâmetros em relação ao modelo FIR.

No caso de implementação adaptativa da lei de controle, uma vantagem prática da utilização de modelos lineares com bases de funções ortonormais (e.g. Laguerre) é a pequena quantidade de informação a priori requerida no processo de identificação: somente uma estimação aproximada do atraso e da constante de tempo dominante do sistema. Como o atraso de transporte também pode ser representado em séries de Laguerre, seu conhecimento preciso não é requerido. A complexidade do modelo (i.e. o número de parâmetros da série) pode ser alterada on-line com um mínimo de perturbação nos parâmetros de ordem mais baixa da série. O modelo a ser identificado é representado em uma estrutura baseada no erro da saída, é linear nos parâmetros e preserva a convexidade do problema de identificação, veja em (Campello et al., n.d.). Ao tornar flexível a escolha da base de funções (estrutura OBF em relação à estrutura FIR), uma seleção adequada aumenta a taxa de convergência dos parâmetros da expansão em séries, diminui a polarização do modelo estimado e a variância do estimador (Heuberger et al., 2005). Estas 
vantagens tornam esta estrutura particularmente apropriada em aplicações industriais de controle adaptativo.

No caso de implementação tipo múltiplos modelos da lei de controle, a mesma base pode ser mantida em todos os modelos lineares que compõe o conjunto limitado de modelos que representa o sistema não linear. Portanto, na equação da lei de controle (12), a primeira linha e, conseqüentemente, o vetor de estados, é igual para todos os modelos. Com isso, a transição entre modelos tende a ser mais suave uma vez que é realizada somente na representação da saída do modelo, isto é, nos coeficientes da base (vetor $c$ do modelo) (Machado, 2007).

\section{CONTROLE PREDITIVO COM MODE- LOS NÃO LINEARES}

Os algoritmos de controle preditivo não linear são aqueles que utilizam modelos não lineares na previsão do comportamento do processo. Conforme discutido na Seção 2, diferentes classes de modelos podem ser utilizados neste contexto. Em particular, a estrutura de modelos não lineares com bases de funções ortonormais pode ser realizada, por exemplo, através de sistemas fuzzy, redes neurais, ou séries de Volterra (veja detalhes em (Campello et al., n.d.)). O caso com Séries de Volterra é apresentado a seguir (Dumont and Fu, 1993; Oliveira et al., 2003).

O modelo OBF-Volterra é dado por:

$$
\begin{aligned}
\boldsymbol{l}(k+1) & =A \boldsymbol{l}(k)+\boldsymbol{b} u(k) \\
y(k) & =\mathcal{H}(\boldsymbol{l}(k))
\end{aligned}
$$

onde o operador $\mathcal{H}$ é:

$$
\begin{gathered}
y(k)=c_{0}+\sum_{i=1}^{n_{1}} c_{i} l_{i}(k)+\sum_{i=1}^{n_{2}} \sum_{l=1}^{n_{2}} c_{i, l} l_{i}(k) l_{l}(k) \\
+\sum_{i=1}^{n_{3}} \sum_{l=1}^{n_{3}} \sum_{m=1}^{n_{3}} c_{i, l, m} l_{i}(k) l_{l}(k) l_{m}(k)+\cdots
\end{gathered}
$$

A equação de previsão, no caso de Séries de Volterra de ordem 2, é:

$$
\begin{gathered}
\hat{y}(k+j \mid k)=c_{0}+\sum_{i=1}^{n_{1}} c_{i} \hat{l}_{i}(k+j \mid k)+ \\
\sum_{i=1}^{n_{2}} \sum_{l=1}^{n_{2}} c_{i, l} \hat{l}_{i}(k+j \mid k) \hat{l}_{l}(k+j \mid k)+e(k)
\end{gathered}
$$

onde $e(k) \triangleq y(k)-\hat{y}(k)$, sendo $y(k)$ e $\hat{y}(k)$ os sinais de saída medido e previsto para o instante de tempo $k$ e $\hat{l}_{i}(k+$ $j \mid k)$ a previsão para os estados do modelo, no instante $k+$ $j$, em função do estado atual $l_{i}(k)$ e dos sinais futuros de controle. Esta formulação para a previsão da saída introduz o sinal de realimentação de saída na malha de controle.

Assim sendo, a lei de controle preditivo não linear, baseada em modelo OBF-Volterra, é definida pelo problema de otimização (6) onde o critério de custo $J_{k}$ é dado pela equação (5). Nota-se que a lei de controle e o critério de custo do algoritmo são idênticos aos apresentados na Seção 3. A diferença recai nas equações de previsão. Portanto, o controle preditivo com modelo OBF linear é um caso particular do controle preditivo com modelo OBF-Volterra, quando somente um núcleo do modelo é utilizado.

A solução ótima do problema (6) são $N_{u}$ valores futuros para o incremento de controle e o sinal efetivamente aplicado ao processo é o primeiro deles. A fim de lidar com limites operacionais, restrições como as dadas por (7) podem ser acrescentadas ao problema (6), que pode ser solucionado através de Programação Quadrática Seqüencial (Sequential Quadratic Programming - SQP) (Edgar and Himmelblau, 1988). Outra possibilidade é a linearização instantânea do modelo a cada instante de amostragem em torno do ponto de operação corrente do sistema (Campello et al., 2003; Campello et al., 2004), de forma que uma lei de controle linear possa ser calculada a cada instante de forma analítica, no caso irrestrito, ou numérica (via programação quadrática), na presença das restrições operacionais supra-mencionadas.

\section{CONTROLE PREDITIVO COM MODE- LOS COM INCERTEZAS}

Os algoritmos de controle preditivo robusto são aqueles que consideram, de maneira explícita no cálculo da lei de controle, as incertezas sobre o modelo do processo. Nesta abordagem, o modelo é descrito não por um, mas sim por um conjunto infinito de modelos lineares onde o processo real pode estar contido.

Modelos OBF (1) com parâmetros incertos são dados por (Oliveira et al., 2000):

$$
\begin{cases}\boldsymbol{l}(k+1) & =A \boldsymbol{l}(k)+\boldsymbol{b} u(k) \\ y(k) & =\boldsymbol{c}(\boldsymbol{\varepsilon})^{T} \boldsymbol{l}(k)\end{cases}
$$

Neste modelo, $\boldsymbol{c}(\varepsilon)$ e $\varepsilon$ são vetores cujos $i$-ésimos elementos são $c_{i}\left(\varepsilon_{i}\right)$ e $\varepsilon_{i}$, respectivamente. $c_{i}\left(\varepsilon_{i}\right)$ são os parâmetros incertos, onde $c_{i, \min } \leq c_{i}(\varepsilon) \leq c_{i, \max }$. Portanto, $\varepsilon$ representa a incerteza do modelo e $\Omega$, com $\varepsilon \subset \Omega$, representa o conjunto de todos os modelos possíveis. A vantagem da utilização de modelos OBF na representação de sistemas incertos 
é que, mantendo a mesma base para todos os modelos, o espaço de incertezas está restrito ao vetor $c$ da representação em espaço de estados (Oliveira et al., 2000). Reescrevendo o modelo (17) no formato incremental, obtém-se:

$$
\begin{cases}\Delta \boldsymbol{l}(k+1) & =A \Delta \boldsymbol{l}(k)+\boldsymbol{b} \Delta u(k) \\ y(k) & =y(k-1)+\boldsymbol{c}(\boldsymbol{\varepsilon})^{T} \Delta \boldsymbol{l}(k)\end{cases}
$$

Utilizando o modelo (18) nas equações de previsão (3) e (4), obtém-se o conjunto de todas as previsões possíveis, i.e., $\hat{y}(k+j \mid k, \varepsilon), \forall \varepsilon \subset \Omega$.

Uma abordagem de controle preditivo robusto, inicialmente apresentada em (Campo and Morari, 1987), baseia a lei de controle na minimização, em relação aos incrementos futuros do sinal de controle, do máximo do critério de custo, em relação ao conjunto de valores possíveis para a saída prevista. A lei de controle é obtida através da otimização on-line de um problema min-max. Esta proposta está baseada em modelos de resposta ao impulso / degrau com coeficientes incertos e o critério de custo é definido utilizando-se uma norma do tipo infinita. Em (Allwright and Papavasiliou, 1992) descreve-se uma estratégia para a resolução do problema min-max cujo objetivo é reduzir a complexidade computacional da lei de controle através da diminuição do número de restrições do problema de otimização. Já em (Zheng and Morari, 1993), propõe-se condições suficientes para garantia da estabilidade do sistema em malha fechada.

A lei de controle preditivo robusta é dada pelo seguinte problema de otimização min-max:

$$
\begin{gathered}
\min _{\Delta \boldsymbol{u}} \max _{\boldsymbol{\varepsilon} \in \Omega} J_{k}(\varepsilon) \\
\text { s.a } \\
\Delta u(k+j \mid k)=0 \quad \forall j=N_{u}, \ldots, N_{y}
\end{gathered}
$$

onde $J_{k}(\varepsilon)$ é o critério de custo, que pode ser:

$$
\begin{aligned}
J_{k, 2}(\varepsilon)= & \sum_{j=1}^{N_{y}}(\hat{y}(k+j \mid k, \boldsymbol{\varepsilon})-w(k+j))^{2} \\
& +\sum_{j=0}^{N_{u}-1} \lambda \Delta u^{2}(k+j \mid k)
\end{aligned}
$$

ou

$$
\begin{gathered}
J_{k, \infty}(\varepsilon)=\max _{j} \mid \hat{y}(k+j / k, \varepsilon)-w(k+j) \\
+\lambda(j) \Delta u^{2}(k+j \mid k) \mid, \quad j=N_{1}, \ldots, N_{y}
\end{gathered}
$$

onde $\lambda(j)$ é o valor de lambda para cada instante de previsão. $\lambda(j)=\lambda$ para $j=0, \ldots, N_{u}-1$ e $\lambda(j)=0$ caso contrário.
Restrições na amplitude e variação do sinal de controle (e.g. equação (7)) também podem ser incorporadas ao problema (19). A solução ótima do problema (19) é obtida assumindo um limite superior $\mu$ para a função de custo, para todo $\varepsilon$ em $\Omega$, isto é:

$$
\begin{aligned}
& \min _{\Delta \boldsymbol{u}} \mu \\
& \text { s.a } \\
& \Delta u(k+j \mid k)=0 \quad \forall j=N_{u}, \ldots, N_{y} \\
& J_{k}(\varepsilon) \leq \mu \quad \forall \varepsilon \in \Omega
\end{aligned}
$$

Neste problema, o número de restrições equivale ao número de modelos pertencentes à família de modelos incertos $\Omega$ que representa o processo. Porém, $\Omega$ é um conjunto com infinitos elementos. Através da utilização de modelos (17), tem-se que a função de custo é convexa em relação aos parâmetros incertos (Oliveira et al., 2000). Isto significa que todos os modelos da família candidatos a solução do problema estão nos vértices de $\Omega$. Uma vez que o número de vértices é finito, a solução numérica do problema (22) é possível pois este passa a ter um número finito de restrições.

A síntese da lei de controle preditivo robusto com modelos OBF preserva os resultados apresentados em (Allwright and Papavasiliou, 1992) e (Zheng and Morari, 1993), i.e. é possível escrever o número de restrições como uma função linear do número de parâmetros incertos e é possível derivar condições suficientes para garantia de estabilidade do sistema em malha fechada (Oliveira et al., 2000).

Devido ao desenvolvimento em séries de funções ortonormais, a utilização do modelo OBF reduz o número de parâmetros incertos do modelo. Assim, o problema de identificação robusta é simplificado (Campello et al., n.d.) e o esforço computacional na solução do problema de otimização minmax é reduzido.

\section{ESTUDO DE CASOS}

Nesta seção, o controle de processos dinâmicos usando algoritmos MBPC com modelos (N)OBF é ilustrado através de dois estudos de caso. O primeiro está relacionado com controle de temperatura e umidade de incubadoras para recém nascidos e envolve controle linear, Seção 3. O segundo é o controle de um reator do tipo CSTR (Continuous Stirred Tank Reactor) e envolve controle não linear, Seção 4.

\subsection{Incubadora para recém - nascidos}

Nesta seção os resultados do controle higro-térmico de incubadoras para recém nascidos utilizando o algoritmo de controle preditivo linear com modelo de Laguerre são analisa- 


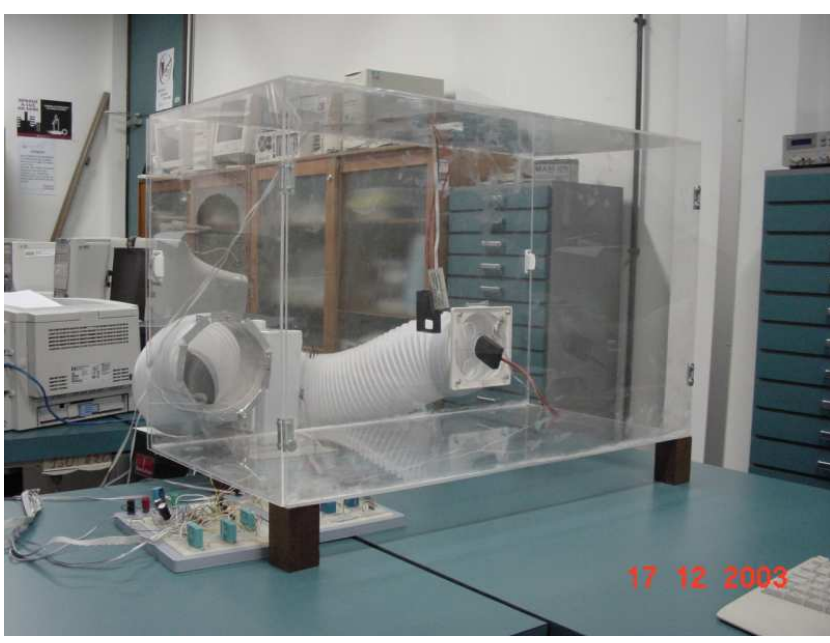

Figura 1: Protótipo de incubadora para recém nascidos.

dos. Primeiramente, o processo dinâmico e o problema de controle são apresentados. Na seqüência, os resultados de controle em tempo real são apresentados e analisados.

Em (Oliveira et al., 2006), um protótipo para testes de sistemas de controle em incubadora é descrito e os principais pontos relacionados com este processo são descritos a seguir. A planta piloto tem as seguintes partes: uma caixa de acrílico $(50 \mathrm{~cm}$ altura $\times 80 \mathrm{~cm}$ largura $\times 40 \mathrm{~cm}$ profundidade); um aquecedor doméstico, um ventilador e um umidificador. $\mathrm{O}$ aquecedor e o umidificador são modificados para permitir controle externo nos quatro níveis de potência permitidos: 0 , $1,2,3$ ou desligado, baixo, médio e máximo. Portanto, o sinal de controle é necessariamente um número inteiro dentro desta faixa. O umidificador é baseado em ultra-som e dutos de ventilação conectam todas as partes citadas e permitem a circulação de ar dentro da incubadora.

A Figura 1 apresenta uma foto da incubadora. Nesta foto, pode-se se ver a caixa acrílica, os dois sensores de temperatura internos e os dois sensores de umidade (duas pequenas caixas pretas), o dispositivo eletrônico (situa-se embaixo da caixa acrílica) e os dutos de ventilação (estão ao lado da incubadora). Alguns orifícios são colocados na lateral da incubadora para proporcionar trocas de ar com o exterior. $\mathrm{O}$ software ambiente para a supervisão e controle digital foi implementado usando o software de instrumentação virtual da LabView, da National Instruments. O período de amostragem é de 0,5 segundos.

A norma técnica Brasileira NBR IEC 60601-2-19 (NBR, 1997) para incubadoras de recém nascidos estabelece que: a temperatura interna da incubadora deve estar dentro da faixa de 32 a $37^{\circ} \mathrm{C}$, a umidade relativa (UR) deve estar no intervalo de 40 a $60 \%$, a sobre-elevação do sinal da temperatura deve ser menor que $2^{\circ} \mathrm{C}$ e, em regime permanente, a temperatura deve oscilar menos que $\pm 0,5^{\circ} \mathrm{C}$ ao redor do sinal de referência.

Assim sendo, o problema é, a partir das condições ambientes, levar a incubadora a condições higro-térmicas próximas de $35^{\circ} \mathrm{C}$ e $55 \%$. Como foi discutido na Seção 3, para síntese da lei de controle preditivo, é necessário um modelo do processo. Baseado em experimentos de resposta ao degrau, o tempo morto (ou atraso) estimado para o modelo de temperatura é de 7 segundos ( $\tau=14$ amostras) e, para o modelo de UR de 10 segundos ( $\tau=20$ amostras). Esses atrasos são incorporados explicitamente no modelo em espaço de estados de Laguerre substituindo $u(k)$ por $u(k-\tau)$ em (1). O pólo da base de Laguerre é selecionado através da obtenção da constante de tempo aproximada do sistema em malha aberta. O valor calculado é de 0,99 (para os modelos de temperatura e de umidade). Portanto, usando um modelo com 4 funções de Laguerre e um estimador baseado em mínimos quadrados, os seguintes coeficientes da série (veja equação (1)) são obtidos:

$$
\begin{gathered}
\left\{c_{i}\right\}_{i=1}^{4}=\{0,43230,16230,08650,0825\} \\
\left\{c_{i}\right\}_{i=1}^{4}=\left\{\begin{array}{llll}
0,3675 & 0,3533 & 0,1840 & 0,0503
\end{array}\right\}
\end{gathered}
$$

para temperatura e UR, respectivamente.

Através do pólo discreto selecionado para as bases de Laguerre (i.e., 0,99), nota-se que o período de amostragem é pequeno em relação à constante de tempo dominante do sistema. Isto indica que modelos adequados podem ser obtidos com valores maiores para o período de amostragem. Entretanto, o nível do sinal de controle assume valores discretos dentro do intervalo $[0,3]$, portanto há necessidade de chaveamento entre níveis de controle para manter a referência em um valor desejado. A utilização de um período de amostragem pequeno tem por objetivo reduzir efeito deste intervalo de tempo entre chaveamentos na amplitude de oscilação do sinal de saída.

Os controladores preditivos de temperatura e UR são construídos conforme apresentado na Seção 3, equação (13). Portanto, a lei de controle tem solução analítica de fácil implementação computacional. Os parâmetros dos controladores de temperatura e UR são: $N_{1}=1, N_{u}=1, \lambda=0 \mathrm{e}$, como $m<N_{u}$, tem-se $m=1$. Em particular, adotou-se $N_{y}=24$ e $N_{y}=30$ para os controladores de temperatura e UR, respectivamente. Uma seleção comum para os parâmetros de MBPC é $N_{1}=N_{u}=1, \lambda=0$ e $N_{y}=10$. Assim sendo, baseado nesta diretriz, os horizontes de previsão estão calculados de modo a ser 10 amostras superior ao atraso de transporte do modelo, que é de 14 e 20 para temperatura e UR, respectivamente. 
O resultado da utilização destes algoritmos no controle em tempo real da incubadora é apresentados nas Figuras 2 e 3. Por meio da Figura 2, pode ser observado que o tempo que

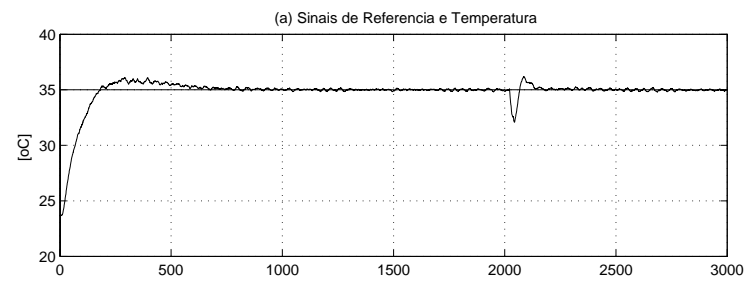

(b) Sinal de Controle - Aquecedor

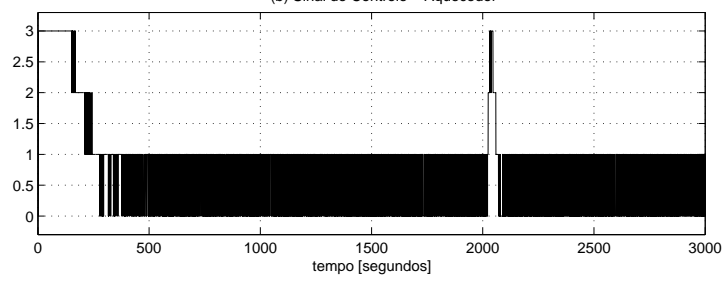

Figura 2: Desempenho do sistema de controle de temperatura com esquema Laguerre-MBPC: (a) sinais de referência e de temperatura em malha fechada; (b) sinal de controle.

o sinal de temperatura leva para atingir a referência é baixo, isto é, próximo a 200 segundos. A sobre-elevação obtida com a lei de controle preditivo é de $1^{\circ} \mathrm{C}$, portando dentro das especificações.

Para o controle de UR, a idéia é compensar, com umidificação, a queda da UR devido ao aquecimento dentro da incubadora. A demora até atingir a umidade de referência do sinal de UR é maior que a observada no sinal de temperatura. Após o instante 2000 segundos, devido a uma perturbação aplicada ao sistema, pode-se observar o restabelecimento das condições de regime permanente pelas duas malhas de controle. No caso, a incubadora é aberta e fechada por instantes, simulando algum procedimento médico a ser realizado.

A principal vantagem da lei de controle preditivo com restrições terminais é a pequena variação da temperatura e da UR ao redor do valor de referência, mesmo com valores inteiros para o sinal de controle. Observe que os sinais estão, aproximadamente, dentro do intervalo $\pm 0,1^{\circ} \mathrm{C}$ e $\pm 0,15 \%$, respectivamente (observe a Figura 4, que apresenta as Figuras 2 e 3 em maiores detalhes).

Portanto, os resultados apresentados mostram que o comportamento do sistema em malha fechada é adequado, principalmente levando em consideração as limitações de hardware (A restrição que limita o sinal de controle em quatro níveis é devida ao fato que a entrada dos circuitos de potência do aquecedor e do umidificador está baseada em dois níveis lógicos). Todos esses fatos validam o algoritmo de controle implementado.
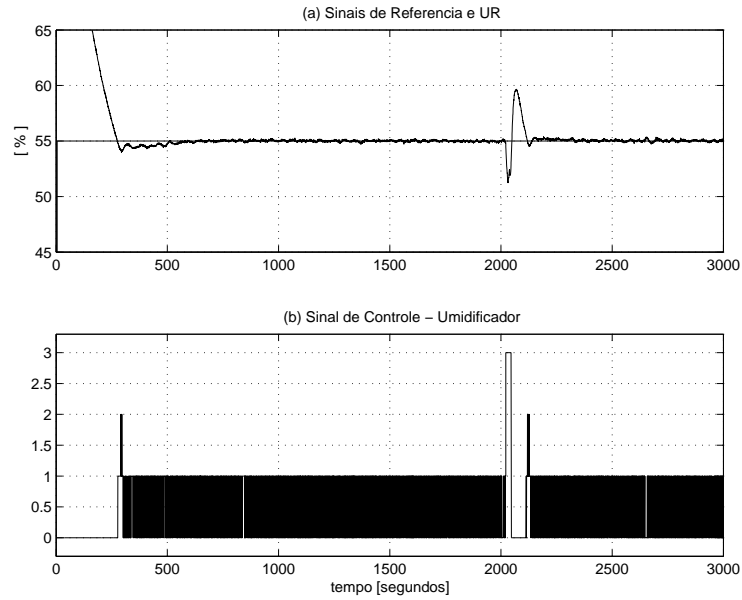

Figura 3: Desempenho do sistema de controle de UR com esquema Laguerre-MBPC: (a) sinais de referência e de umidade em malha fechada; (b) sinal de controle.

\subsection{Unidade CSTR}

O problema aqui analisado é o controle de um reator CSTR. Este processo, descrito em (Doyle et al., 1995), é uma polimerização isotérmica usando tolueno como solvente. $\mathrm{O}$ peso molecular médio da reação é controlado através da manipulação do fluxo do iniciador. O conjunto de equações diferenciais que relaciona os sinais de entrada e saída do processo é apresentado em (Campello et al., n.d.), a primeira parte deste trabalho.

Para este exemplo, utiliza-se um modelo OBF-Volterra com base de Laguerre (pólo em $p=0,85$ para um período de amostragem de $\Delta t=0.03$ horas, do segurador de ordem zero do controlador) e estrutura com $n_{1}=5$ e $n_{2}=3$ para as partes linear e quadrática, respectivamente:

$$
y(k)=\sum_{i=1}^{5} c_{i} l_{i}(k)+\sum_{i=1}^{3} \sum_{l=1}^{3} c_{i, l} l_{i}(k) l_{l}(k)
$$

onde os parâmetros $c_{i}$ e $c_{i, l}$ são dados por:

$$
\left[\begin{array}{c}
-0,1021 \\
-0,0415 \\
0,0294 \\
-0,0120 \\
0,0051
\end{array}\right] \text { e } \quad\left[\begin{array}{ccc}
0,0152 & 0,0050 & -0,0014 \\
0,0050 & 0,0015 & -0,0012 \\
-0,0014 & -0,0012 & 0,0015
\end{array}\right]
$$

respectivamente. Sabe-se que a capacidade de aproximação do processo pelo modelo OBF-Volterra depende do pólo e do 

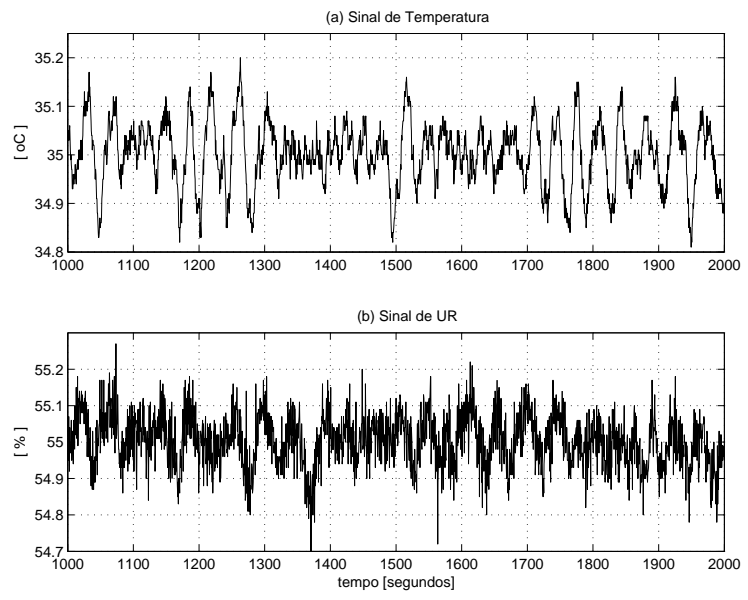

Figura 4: Detalhe das Figuras 2 e 3: (a) sinal de temperatura; (b) sinal de umidade.

número de funções da base. Na Tabela 1 tem-se quatro situações diferentes, sendo uma delas a utilizada neste exemplo. Nota-se que o modelo com pólo 0.85 apresenta melhor aproximação que aquele com pólo em 0.35 (caso 2 em relação ao caso 1), uma vez que o primeiro é mais próximo do valor ótimo calculado em (Campello et al., n.d.). Nota-se também que modelos cuja base da parte quadrática possui mais funções apresentam melhor aproximação (caso 2 em relação ao 4); entretanto, valores elevados de funções na base não trazem necessariamente uma melhora significativa na aproximação do modelo (caso 3 em relação ao 2). Mais além, é importante destacar que modelos mais precisos sob um determinado critério não levam necessariamente a um melhor desempenho do sistema em malha fechada também segundo algum critério. Formas com as quais a precisão do modelo pode influenciar o desempenho da malha e como imprecisões no modelo podem ser compensadas pelo sistema de controle são abordadas, por exemplo, em (Aguirre and Tôrres, 2000).

Tabela 1: Comparação entre diferentes estruturas de modelo. O Erro Quadrático Médio (EQM) deve ser multiplicado por $\times 10^{-6}$ e o erro é referente ao máximo valor absoluto do erro durante a previsão.

\begin{tabular}{|c|c|c|c|c|c|}
\hline caso & pólo & $n_{1}$ & $n_{2}$ & EQM & erro \\
\hline 1 & 0,35 & 5 & 3 & 81,2 & $5 \times 10^{-2}$ \\
\hline 2 & 0,85 & 5 & 3 & 3,51 & $0,55 \times 10^{-2}$ \\
\hline 3 & 0,85 & 10 & 5 & 3,04 & $0,45 \times 10^{-2}$ \\
\hline 4 & 0,85 & 3 & 1 & 9,12 & $1 \times 10^{-2}$ \\
\hline
\end{tabular}

Neste exemplo, o modelo OBF-Volterra (caso 2) contém 11 parâmetros (uma vez que $c_{i, j}=c_{j, i}$ ), contra 136 a 496 parâmetros para um modelo de Volterra NFIR (i.e. sem desenvolvimento em séries ortonormais) (Maner et al., 1996). Esta parcimônia na representação do processo simplifica o procedimento de identificação e a implementação da lei de controle preditivo.

Os parâmetros da lei de controle são: $N_{y}=10, N_{u}=1$, $\lambda=0$ e $m=1$. A diretriz para a seleção destes horizontes é a mesma do exemplo anterior. O algoritmo utilizado na otimização da lei de controle (6) é o Sequencial Quadratic Programming - SQP. O comportamento do sistema em malha fechada para um sinal de referência começando em 25000,5 (a condição nominal de operação (Campello et al., n.d.)) e assumindo os valores de 37500, 25000, 40000, 30000 é apresentado na Figura 5. Nesse caso, o desempenho do sistema em malha fechada é adequado, devido especialmente à boa qualidade do modelo.

O desempenho do sistema em malha fechada, em termos da sobre-elevação, é ilustrado na Tabela 2. A aproximação do modelo para diferentes valores dos pólos e número de funções é ilustrada na Tabela 1. Nestas tabelas, pode-se notar o efeito da localização do pólo e do número de funções da base na aproximação do modelo do processo e no conseqüente desempenho do sistema. Como era esperado, o desempenho do sistema em malha fechada, neste caso, representado por uma menor sobre-elevação em resposta a um degrau no sentido positivo, melhora quando a qualidade do modelo (sob o ponto de vista de um compromisso entre aproximação via
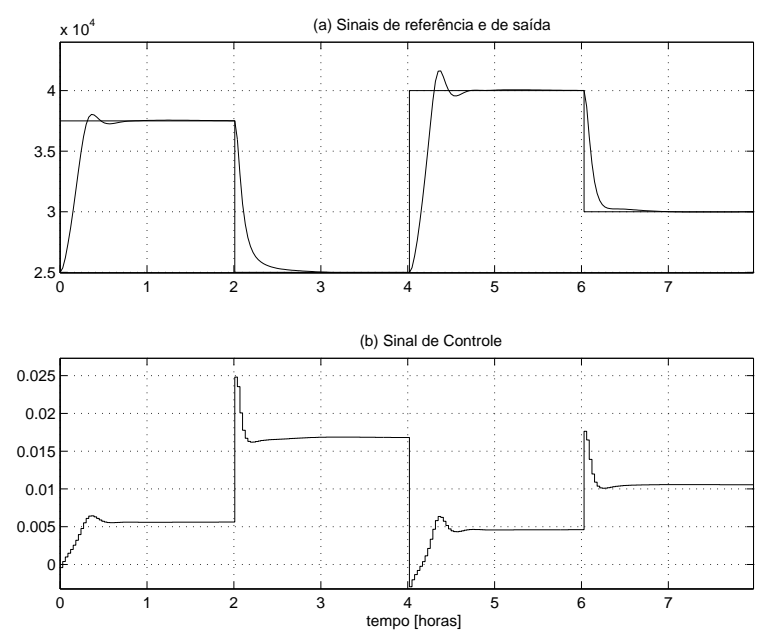

Figura 5: Desempenho do sistema em malha fechada usando controle preditivo não linear baseado em modelo OBFVolterra. (a) sinais de referência e de saída; (b) sinal de controle.

EQM e número de parâmetros) também melhora. 
Tabela 2: Comparação de desempenho do sistema em malha fechada. Sobre-elevação em relação à amplitude do degrau aplicado.

\begin{tabular}{|c|c|c|}
\hline caso & $1^{\text {o. }}$ degrau & $2^{\text {o. }}$ degrau \\
\hline 1 & $31,2 \%$ & $44,7 \%$ \\
\hline 2 & $4 \%$ & $10 \%$ \\
\hline 3 & $8,8 \%$ & $16,7 \%$ \\
\hline 4 & $9,6 \%$ & $21,3 \%$ \\
\hline
\end{tabular}

\section{CONCLUSÕES}

Este artigo apresentou a aplicação, características e vantagens de bases de funções ortonormais no contexto de sistemas de controle preditivo. Inicialmente, o problema da seleção de modelos em controladores foi revista. Na seqüência, a aplicação de modelos (N)OBF em controladores lineares, não lineares e com incertezas (caso robusto) foi descrita. Dois casos de estudo foram apresentados, um envolvendo o controle de temperatura e umidade de uma incubadora real para recém-nascidos e o outro envolvendo o controle do peso molecular do produto na saída de um reator de polimerização isotérmica simulado.

\section{AGRADECIMENTOS}

$\mathrm{O}$ segundo e terceiro autores agradecem ao Conselho Nacional de Desenvolvimento Científico e Tecnológico CNPq (processos 307554/2003-1 e 305032/2003-8, respectivamente).

\section{REFERÊNCIAS}

Aguirre, L. A. (2000). Introdução à Identificação de Sistemas: Técnicas Lineares e Não Linares Aplicadas a Sistemas Reais, Editora UFMG.

Aguirre, L. A. and Tôrres, L. A. B. (2000). Control of nonlinear dynamics: Where do models fit in?, International Journal of Bifurcation and Chaos 10(3): 667-681.

Allgower, F. and Zheng, A. (2000). Nonlinear model predictive control, Progress in Systems and Control Theory, Birkhauser Verlag, Switzerland.

Allwright, J. C. and Papavasiliou, G. C. (1992). On linear programming and robust model-predictive control using impulse-responses, Systems \& Control Letters 18: 159-164.
Bemporad, A. and Morari, M. (1999). Control of systems integrating logic dynamics and constrains, Automatica 35(2): 407-427.

Bemporad, A., Morari, M., Dua, V. and Pistikopoulos, E. N. (2000). The explicit linear quadratic regulator for constrained systems, Automatica 38(1): 3-20.

Biegler, L. T. (1989). On solving the fundamental control problem in the presence of uncertainty : A mathematical programming approach, in D. M. Prett, C. E. Garcia and B. L. Ramaker (eds), The Second Shell Process Control Workshop, Butterworths, pp. 149-180.

Bitmead, R. R., Gevers, M. and Wertz, V. (1990). Adaptive Optimal Control: The Thinking Man's GPC, Prentice Hall.

Camacho, E. (1993). Constrained generalized predictive control, IEEE Trans. on Automatic Control 38(2): 327-332.

Camacho, E. F. and Bordons, C. (1999). Model Predictive Control in Process Industry, 2 edn, Springer Verlag.

Campello, R. J. G. B., Meleiro, L. A. C. and Amaral, W. C. (2004). Control of a bioprocess using orthonormal basis function fuzzy models, Proc. 13th IEEE Internat. Conference on Fuzzy Systems, Budapest/Hungary, pp. 801806.

Campello, R. J. G. B. and Oliveira, G. H. C. (2007). Modelos não lineares, in L. A. Aguirre, A. P. Alves da Silva, M. F. M. Campos and W. C. Amaral (eds), Enciclopédia de Automática, Vol. 3 (Cap. 4), Edgard Blücher.

Campello, R. J. G. B., Oliveira, G. H. C. and Amaral, W. C. (n.d.). Identificação e controle de processos via desenvolvimentos em séries ortonormais. Parte A: Identificação, Controle \& Automação .

Campello, R. J. G. B., Von Zuben, F. J., Amaral, W. C., Meleiro, L. A. C. and Maciel Filho, R. (2003). Hierarchical fuzzy models within the framework of orthonormal basis functions and their application to bioprocess control, Chemical Engineering Science 58: 4259-4270.

Campo, P. J. and Morari, M. (1986). Infinity norm formulation of model predictive control problems, Proc. of the IEEE American Control Conference, pp. 339-343.

Campo, P. J. and Morari, M. (1987). Robust model predictive control, Proc. of the IEEE American Control Conference, Minneapolis/USA, pp. 1021-1026.

Chow, C. M., Kuznetsov, A. G. and Clarke, D. W. (1995). Using multiple models in predictive control, Proc. of the 3rd European Control Conference, pp. 1732-1737. 
Clarke, D. W. (ed.) (1994). Advances in Model Based Predictive Control, Oxford University Press.

Clarke, D. W. and Mohtadi, C. (1989). Properties of generalized predictive control, Automatica 25(6): 859-875.

Clarke, D. W., Mohtadi, C. and Tuffs, P. (1987). Generalized predictive control - Part 1 : The basic algorithm - Part 2 : Extensions and interpretations, Automatica 23(2): 149-160.

Clarke, D. W. and Scattolini, R. (1991). Constrained receding horizon predictive control, IEE Proc. D 138(4): 347-354.

Cuthrell, J. E., Rivera, D. E., Schmidt, W. J. and Vegeais, J. A. (1989). Solution to the Shell standard control problem, in D. M. Prett, C. E. Garcia and B. L. Ramaker (eds), The Second Shell Process Control Workshop, Butterworths, pp. 27-58.

Cutler, C. R. and Ramaker, B. C. (1980). Dynamic matrix control - A computer control algorithm, Proc. of the IEEE American Control Conference, San Francisco/USA.

Cuzzola, F. C., Geromel, J. C. and Morari, M. (2002). An improved approach for constrained robust model predictive control, Automatica 38: 1183-1189.

de Keyser, R. M. and Cuawenberghe, A. R. V. (1985). Extended prediction self-adaptive control, IFAC Symp. on Identification and System Parameter Estimation, pp. 1317-1322.

de Keyser, R. M., de Velde, P. A. V. and Dumortier, F. (1988). A comparative study of self adaptive long-range control methods, Automatica 24(2): 149-163.

Demircioglu, H. and Clarke, D. W. (1993). Generalized predictive control with end-point state weighting, IEE Proc.-D 140(4): 275-282.

den Hof, P. M. J. V., Heuberger, P. S. C. and Bokor, J. (1995). System identification with generalized orthonormal basis functions, Automatica 31(12): 1821-1834.

Ding., B., Xi, Y. and Li, S. (2004). A synthesis approach of on-line constrained robust model predictive control, Automatica 40: 163-167.

Dolye, F. J., Pearson, R. K. and Ogunnaike, B. A. (2001). Identification and Control using Volterra Models, Springer.

Doyle, F. J., Ogunnaike, B. A. and Pearson, R. K. (1995). Nonlinear model-based control using second-order Volterra models, Automatica 31(5): 697-714.
Dumont, G. A., Elnaggar, A. and Elshafei, A. (1993). Adaptive predictive control of systems with time-varying time delay, International Journal of Adaptive Control and Signal Processing 7: 91-101.

Dumont, G. A. and Fu, Y. (1993). Non-linear adaptive control via Laguerre expansion of Volterra kernels, International Journal of Adaptive Control and Signal Processing 7: 367-382.

Dumont, G. A., Zervos, C. and Pageau, G. L. (1990). Laguerre-based adaptive control of $\mathrm{pH}$ in an industrial bleach plant extraction stage, Automatica 26(4): 781787.

Edgar, T. F. and Himmelblau, D. M. E. (1988). Optimization of Chemical Processes, McGraw-Hill.

Elshafei, A., Dumont, G. A. and Elnaggar, A. (1994). Adaptive GPC based on Laguerre-filters modelling, Automatica 30(12): 1913-1920.

Finn, C., Wahlberg, B. and Ydstie, B. E. (1993). Constrained predictive control using orthogonal expansions, AIChE Journal 39(11): 1810-1826.

Garcia, C. E., Prett, D. M. and Morari, M. (1989). Model predictive control: Theory and practice - A Survey, $A u$ tomatica 25(3): 335-348.

Gutierrez, A. J. and Camacho, E. F. (1995). Robust adaptive control for processes with bounded uncertainties, Proc. of 3rd European Control Conference, Vol. 2, Roma/Italy, pp. 1295-1300.

Haber, R., Bars, R. and Lengvel, O. (1998). Long-range predictive control of parametric hammerstein model, Proc. of the IFAC NOLCOS, pp. 434-439.

Heemels, W. P. M. H., de Schutter, B. and Bemporad, A. (2001). Equivalence of hybrid dynamical models, $A u$ tomatica 37: 1085-1091.

Heuberger, P., den Hof, P. V. and Wahlberg, B. (eds) (2005). Modelling and Identification with Rational Orthogonal Basis Functions, Springer Verlag.

Kothare, M. V., Balakrishnan, V. and Morari, M. (1996). Robust constrained model predictive control using linear matrix inequalities, Automatica 32(10): 1361-1379.

Labinaz, G., Bayoumi, M. M. and Rudie, K. (1997). A survey of modeling and control of hybrid systems, Annual Reviews in Control 21: 79-92.

Lee, J. H., Morari, M. and Garcia, C. E. (1994). State-space interpretation of model predictive control, Automatica 30(4): 707-717. 
Lennartson, B., Egardt, B. and Tittus, M. (1994). Hybrid systems in process control, Proc. of the IEEE Conference on Decision and Control, pp. 3587-3592.

Leva, A. and Scattolini, R. (1993). Predictive control with terminal constraints, Proc. of European Control Conference, pp. 932-936.

Li, S., Lim, K. Y. and Fisher, D. G. (1989). A state-space formulation for model predictive control, AIChE Journal 35: $241-249$.

Ljung, L. (1999). System Identification: Theory for the user, 2 edn, Prenctice Hall, Inc.

Machado, J. B. (2007). Modelagem e controle preditivo utilizando multi-modelos, Master's thesis, DCA/FEEC/UNICAMP, Campinas/SP, Brasil.

Malacalza, D. E. and Jr., J. M. G. S. (2005). Aplicação de controle preditivo baseado em modelos a sistemas mistos lógicos-dinâmicos, VII SBAI.

Maner, B. R., III, F. J. D., Ogunnaike, B. A. and Pearson, R. K. (1996). Nonlinear model predictive control of a simulated multivariable polymerization reactor using second-order Volterra models, Automatica 32(9): 1285-1301.

Mayne, D. Q., Rawlings, J. B., Rao, C. V. and Scokaert, P. O. M. (2000). Constrained model predictive control: stability and optimality, Automatica 36: 789-814.

Moreira, V. D. (2006). Controle Preditivo Robusto de Sistemas Hibridos Incertos Integrando Restrições, Lógica e Dinâmica Baseada em Séries de Funções Ortonormais, $\mathrm{PhD}$ thesis, DCA/FEEC/UNICAMP.

Mosca, E. and Zhang, J. (1992). Stable redesign of predictive control, Automatica 28(6): 1229-1233.

NBR (1997). Electro medical equipments, Part 2: Prescriptions for safety in neonate incubators (in Portuguese), NBR IEC 60601-2-19 edn, Brazilian Technical Norms Association, Rio de Janeiro.

Nelles, O. (2001). Nonlinear System Identification, SpringerVerlag.

Norquay, S., Palazoglu, A. and Romagnoli, J. A. (1999). Aplication of wiener model predictive control (WMPC), IEEE Trans. on Control System Technology 7(4): 437-445.

Oliveira, G. H. C., Amaral, W. C. and Favier, G. (1999). Adaptive predictive controller using orthonormal series functions, Applied Mathematics and Computer Science 9(1): 175-192.
Oliveira, G. H. C., Amaral, W. C., Favier, G. and Dumont, G. (2000). Constrained robust predictive controller for uncertain processes modeled by orthonormal series functions, Automatica 36(4): 563-572.

Oliveira, G. H. C., Amaral, W. C. and Latawiec, K. (2003). CRHPC using volterra models and orthonormal basis functions: An application to CSTR plants, Proc. of the IEEE Conference on Control Aplications, Vol. 1, Istanbul/Turkey, pp. 718-723.

Oliveira, G. H. C., Amorim, M. F. and Pacholok, C. (2006). A real-time predictive scheme for controlling hygrothermal conditions of neonate incubators, Proc. of the IFAC Symposium on Modelling and Control of Biomedical Systems, Reims/France.

Oliveira, J. V. and Lemos, J. M. (1995). Long-range predictive adaptive fuzzy relational control, Fuzzy Sets and Systems 70: 337-357.

Ordys, A. W. and Clarke, D. W. (1993). A state-space description for GPC controllers, Int. J. Systems Science 24(9): 1727-1744.

Patwardhan, S. C., Manuja, S., Narasimhan, S. and Shah, S. L. (2006). From data to diagnosis and control using generalized orthonormal basis filters. Part II: Model predictive and fault tolerant control, Journal of Process Control 16: 157-175.

Patwardhan, S. C. and Shah, S. L. (2005). From data to diagnosis and control using generalized orthonormal basis filters. Part I: Development of state observers, Journal of Process Control 15: 819-835.

Prett, D. M. and Garcia, C. E. (1988). Fundamental Process Control, Butterworths Inc.

Prett, D. M. and Garcia, C. E. (1989). The Second Shell Process Control Workshop: Solutions to the Shell Standard Control Problem, Butterworths Inc.

Qina, S. J. and Badgwell, T. A. (2003). A survey of industrial model predictive control technology, Control Engineering Practice 11: 733-764.

Richalet, J., Rault, A., Testud, J. L. and Papon, J. (1978). Model predictive heuristic control : Applications to industrial process, Automatica 14(5): 413-428.

Rossiter, J. A. (2003). Model-based Predictive Control: A practical approach, CRC Press.

Saint-Donat, J. (1991). Neural based model predictive control, International Journal of Control 54(6): 14531468 . 
Soeterboek, R. (1992). Predictive Control : A Unified Approach, Prentice Hall International.

Trierweiler, J. O. and Secchi, A. R. (2000). Exploring the potientiality of using multiple model approach in nonlinear model predictive control, in F. Allgower and A. Zheng (eds), Nonlinear Model Predictive Control, Progress in Systems and Control Theory, Birkhauser Verlag, pp. 191-203.

Tsang, T. T. C. and Clarke, D. W. (1988). Generalized predictive control with input constraints, IEE Proc. - D 132(3): 100-110.

Wahlberg, B. and Makila, P. M. (1996). On approximation of stable linear dynamical systems using Laguerre and Kautz functions, Automatica 32(5): 693-708.

Wellers, M. and Rake, H. (2000). Nonlinear Model Predictive Control, Birkhauser Verlag, chapter Nonlinear Model Predictive Control Based on Stable Wiener and Hammerstein Models, pp. 357-368.

Ydstie, B. E. (1984). Extended horizon adaptive control, Proc. of 9th IFAC World Congress, Budapest/Hungria.

Zervos, C. C. and Dumont, G. A. (1988). Deterministic adaptive control based on Laguerre series representation, International Journal of Control 48(6): 2333-2359.

Zheng, Z. Q. and Morari, M. (1993). Robust stability of constrained model predictive control, Proc. of the IEEE American Control Conference, San Francisco/USA, pp. 379-383.

Zhu, Q. M., Warwick, K. and Douce, J. L. (1991). Adaptive general predictive controller for nonlinear systems, IEE Proc.-D 138(1): 33-40. 